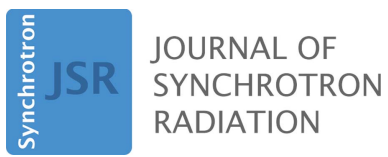

ISSN 1600-5775

Received 31 March 2021

Accepted 15 September 2021

Edited by M. Yabashi, RIKEN SPring-8 Center, Japan

Keywords: micro-CT; phase-contrast CT; low-temperature $\mathrm{CT}$; time-resolved topography; Ge double-crystal monochromator; operando topography.

\section{Advanced X-ray imaging at beamline 07 of the SAGA Light Source}

\author{
Akio Yoneyama, ${ }^{\mathrm{a}, \mathrm{b}, \mathrm{c} *}$ Satoshi Takeya, ${ }^{\mathrm{d}}$ Thet Thet Lwin, ${ }^{\mathrm{b}}$ Daiko Takamatsu, \\ Rika Baba, ${ }^{\mathrm{c}}$ Kumiko Konishi, ${ }^{\mathrm{c}}$ Ryusei Fujita, ${ }^{\mathrm{c}}$ Keisuke Kobayashi, ${ }^{\mathrm{c}}$ Akio Shima, ${ }^{\mathrm{c}}$ \\ Masahide Kawamoto, ${ }^{a}$ Hiroyuki Setoyama, ${ }^{a}$ Kotaro Ishiji ${ }^{\mathrm{a}}$ and Yoshiki Seno ${ }^{\mathrm{a}}$

\footnotetext{
aBeamline Group, SAGA Light Source, 8-7 Yayoigaoka, Tosu, Saga 841-0005, Japan, ${ }^{\mathbf{b}}$ Allied Health Sciences, Kitasato University, 1-15-1 Kitasato, Minamiku, Sagamihara, Kanagawa 252-0373, Japan, ${ }^{\mathbf{c}}$ Research and Development Group, Hitachi Ltd, 1-280 Higashi-koigakubo, Kokubunji, Tokyo 185-8601, Japan, and 'National Metrology Institute of Japan, National Institute of Advanced Industrial Science and Technology (AIST), Central 5, 1-1-1 Higashi, Tukuba, Ibaraki 305-8565, Japan. *Correspondence e-mail: yoneyama@saga-Is.jp
}

The SAGA Light Source provides X-ray imaging resources based on highintensity synchrotron radiation (SR) emitted from the superconducting wiggler at beamline 07 (BL07). By combining quasi-monochromatic SR obtained by the newly installed water-cooled metal filter and monochromatic SR selected by a Ge double-crystal monochromator (DCM) with high-resolution lens-coupled $\mathrm{X}$-ray imagers, fast and low-dose micro-computed tomography (CT), fast phasecontrast CT using grating-based X-ray interferometry, and 2D micro-X-ray absorption fine structure analysis can be performed. In addition, by combining monochromatic SR obtained by a Si DCM with large-area fiber-coupled X-ray imagers, high-sensitivity phase-contrast CT using crystal-based X-ray interferometry can be performed. Low-temperature CT can be performed using the newly installed cryogenic system, and time-resolved analysis of the crystallinity of semiconductor devices in operation can be performed using a time-resolved topography system. The details of each instrument and imaging method, together with exemplary measurements, are presented.

\section{Introduction}

Synchrotron radiation (SR) is several orders of magnitude brighter than X-rays from conventional X-ray tubes, and its high brilliance has been used for X-ray imaging in various fields such as biomedicine, materials science, archeology and geochemistry. The SAGA Light Source facility (stored energy $1.4 \mathrm{GeV}$, maximum ring current $300 \mathrm{~mA}$ ) in Saga Prefecture, Japan, was established in 2006 as a source of SR dedicated to a wide range of experiments. SR from a bending magnet (BL15) and from a superconducting wiggler (BL07) (Sumitani et al., 2013) has been used to perform computed tomography (CT) and diffraction-enhanced phase-contrast CT experiments. $\mathrm{X}$-ray fluorescence microscopy using an X-ray beam focused by a Fresnel zone plate (FZP) has also been conducted as well (Sumitani et al., 2011). Furthermore, imaging methods such as the sub-pixel-shift method that uses photon-counting detectors to improve the spatial resolution (Yoneyama, Baba, Sumitani et al., 2015) and dual-energy CT to calculate the effective atomic number have been developed for use at the site. X-ray topography equipment using X-ray film has also been made available (Ishiji et al., 2011).

We have been developing advanced X-ray imaging techniques at BL07 to enable researchers to conduct non-destructive investigations. In particular, we have developed a fast phase- 


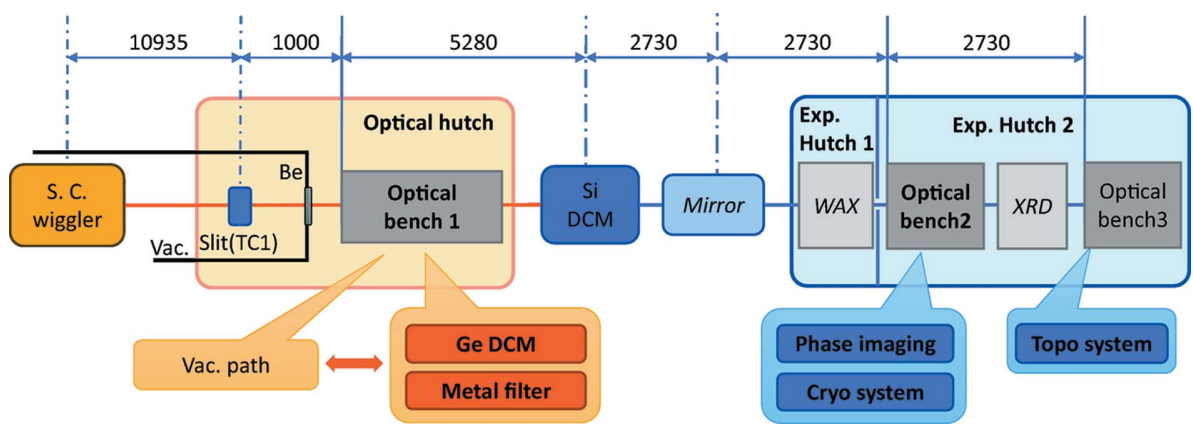

Figure 1

Layout of BL07 at SAGA Light Source and newly installed instrumentation (bold).

without higher-order effects, which is especially useful for XAFS measurements, can be used in the experimental hutches. Note that a vacuum path has been installed in the optical hutch to reduce flux loss caused by X-ray scattering in the air when SR after passing the Si DCM is used.

The optical hutch has been used to perform white SR irradiation experiments on plant seeds (Sakamoto et al., 2019). In particular, SR irradiation was used to genetically modify various flowers that blossomed in unnatural

contrast CT system using diffraction-enhanced imaging. The system has been used to make $3 \mathrm{D}$ observations within a 10 min scanning time (Yoneyama et al., 2020). In addition, we have developed a scanning X-ray fluorescence microscopy (SXFM) system using total-reflection mirrors and white SR. This system has been used to map the distributions of elements such as $\mathrm{S}, \mathrm{Cl}, \mathrm{K}$ and $\mathrm{Ca}$ in plant seeds (Yoneyama \& Kawamoto, 2020). We have also developed high-resolution lens-coupled X-ray imagers using optimized phosphor (Yoneyama et al., 2021). Most recently, we developed a watercooled metal filter, a Ge double-crystal monochromator (DCM), phase-contrast CT systems using a grating-based and a crystal-based X-ray interferometer, a cold-nitrogen-gas cryogenic system and a time-resolved X-ray topography system.

As a result of these developments, the following forms of X-ray imaging can now be performed at the SAGA Light Source: (i) fast high-spatial-resolution CT (fast micro-CT) using high-intensity quasi-monochromatic (pink) SR, (ii) lowdose micro-CT, (iii) micro-2D X-ray absorption fine structure (XAFS) analysis, (iv) fast phase-contrast CT, (v) high-sensitivity phase-contrast CT, (vi) low-temperature CT and (vii) time-resolved analysis of crystallinity of semiconductor devices in operation. In this article, we report on the experimental instruments and imaging methods, as well as some exemplary applications of the advanced X-ray imaging methods carried out at BL07 of the SAGA Light Source.

\section{Beamline layout, experimental instrumentation and SR properties}

\subsection{Outline}

Beamline 07 (BL07) of the SAGA Light Source consists mainly of a superconducting magnet wiggler, an optical hutch, a Si DCM (Sumitani et al., 2013), a total-reflection focusing mirror, and experimental hutches 1 and 2, as shown in Fig. 1 (Kawamoto et al., 2010). White SR emitted from the wiggler and shaped by a slit consisting of water-cooled Ta blades can be used directly in the optical hutch. Parallel SR monochromated by the Si DCM using Si (220) or Si (111) diffraction can be employed in experimental hutches 1 and 2 . In addition, by using the focusing mirror, focused monochromatic SR colors. Experimental hutch 1 has wide-angle X-ray diffraction (WAXD) and wide-angle X-ray scattering (WAXS) (Ohtani et al., 2017) equipment. It has been used to perform protein crystallography (PX) (Arai et al., 2015) measurements. Experimental hutch 2 has a six-axis diffractometer and an XAFS measurement system which are installed in a tandem arrangement, and it has been used to perform X-ray diffraction (XRD), XAFS (Okajima et al., 2018) and X-ray fluorescence analysis (XRF) (Tabata \& Ueda, 2017) experiments using monochromatic SR above $10 \mathrm{keV}$.

\subsection{Upgrade of X-ray optics}

2.2.1. Compact DCM using Ge (111) diffraction. A portable compact DCM using Ge (111) diffraction was developed and installed in the optical hutch. As shown in Fig. 2(a), the compact Ge DCM consists of the first crystal and its positioning tables (purple), the second crystal and its positioning tables (green), and the main rotational mount of the crystal-positioning tables and its positioning tables (light blue). The main rotational-axis angle (Bragg angle) ranges from 5 to $18.5^{\circ}$, corresponding to 7 to $20 \mathrm{keV}$. The beam size is $20 \mathrm{~mm} \times 2 \mathrm{~mm}$, which is large enough for micro-CT and micro-XAFS. Since the compact DCM must be removed from the SR path in order to use the downstream experimental hutches, it uses small Ge crystals $(30 \mathrm{~mm} \times 35 \mathrm{~mm})$, which reduce its length to less than $500 \mathrm{~mm}$ for easy transport. Moreover, the main chamber of the DCM substitutes He gas for a vacuum, and the chamber itself is made of acrylic to reduce its weight $(20 \mathrm{~kg})$. Owing to the short distance from the

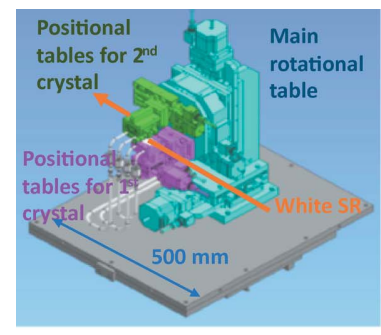

(a)

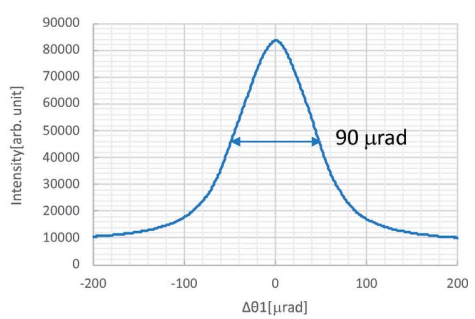

(b)
Figure 2

(a) Schematic view of the compact Ge DCM and (b) rocking curve obtained by rotating the first crystal. 
Table 1

Main specifications of X-ray imagers at SAGA Light Source.

\begin{tabular}{|c|c|c|c|c|c|c|}
\hline & Manufacturer and name & Type & Field of view (mm) & Pixel size $(\mu \mathrm{m})$ & Pixel number & Phosphor \\
\hline 1 & SAGA-LS, Kenvy 1 & Lens coupling & $\begin{array}{l}3.3 \times 2.8 \text { for } \times 5 \text { lens } \\
1.6 \times 1.4 \text { for } \times 10 \text { lens }\end{array}$ & $\begin{array}{l}1.3 \text { for } \times 5 \text { lens } \\
0.65 \text { for } \times 10 \text { lens }\end{array}$ & $2560 \times 2160$ & $\mathrm{Lu}_{3} \mathrm{Al}_{5} \mathrm{O}_{12}: \mathrm{Ce}(\mathrm{LuAG})$ \\
\hline 2 & SAGA-LS, Kenvy 2 & Lens coupling & $\begin{array}{l}2.6 \times 2.6 \text { for } \times 5 \text { lens } \\
1.3 \times 1.3 \text { for } \times 10 \text { lens }\end{array}$ & $\begin{array}{l}1.3 \text { for } \times 5 \text { lens } \\
0.65 \text { for } \times 10 \text { lens }\end{array}$ & $2048 \times 2048$ & CsI:Tl (CsI) \\
\hline 3 & Photonic Science, VHR & Fiber coupling & $50 \times 35$ & 12.5 & $4008 \times 2650$ & $\mathrm{Gd}_{2} \mathrm{O}_{2} \mathrm{~S}: \mathrm{Tb}(\mathrm{GOS})$ \\
\hline 4 & Andor, Zyla 5.5 HF & Fiber coupling & $16 \times 13$ & 6.5 & $2560 \times 2160$ & CsI:Tl (CsI) \\
\hline
\end{tabular}

wiggler $(\sim 12 \mathrm{~m})$ and the wide bandwidth (diffraction width) of Ge (111), high-intensity monochromatic SR can be used.

Fig. 2(b) shows a rocking curve obtained by rotating the first crystal. The full width at half-maximum (FWHM) is $90 \mu \mathrm{rad}$, which is consistent with the FWHM calculated from the Ge diffraction width and the vertical beam divergence of the SR.

2.2.2. Water-cooled metal filter. A water-cooled metal filter is installed in the optical hutch to narrow the energy band and optimize the peak energy of white SR depending on the samples' thickness and composition. A combination of metal filters such as aluminium, copper, nickel and zirconium of 0.1, 0.2 and $0.5 \mathrm{~mm}$ thickness can be chosen.

\subsection{SR flux}

The photon fluxes of the monochromatic SR obtained by the Ge and Si DCMs are shown in Fig. 3. The flux was calculated from the detected ionization current of the ion chamber (OKEN S-1329) and the gas composition (50\% $\mathrm{N}_{2}$ and $50 \%$ Ar) by using Hephaestus software (Ravel \& Newville, 2005). Since the top-up operation is not supported at the SAGA Light Source and, hence, the photon flux decreases with time, the flux was normalized at a ring current of $200 \mathrm{~mA}$, which is $2 / 3$ of the maximum stored current. Moreover, a four-blade slit $(Q$ slit $)$ with a $1 \mathrm{~mm} \times 1 \mathrm{~mm}$ opening was set upstream of the ion chamber to normalize the beam size.

The results in the figure show that the photon flux using the Ge DCM amounts to $2 \times 10^{9}$ photons s $\mathrm{sm}^{-1}$ at $10 \mathrm{keV}$,

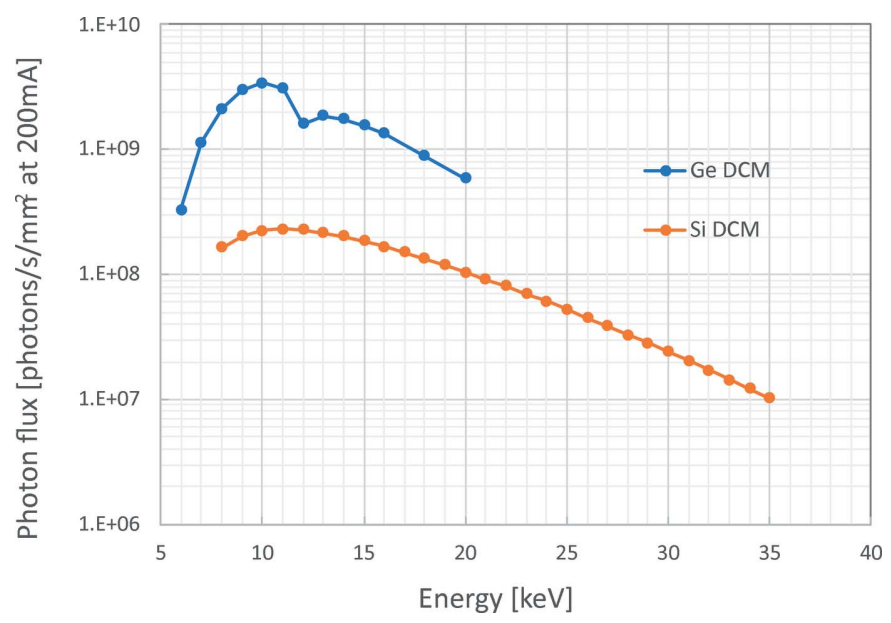

Figure 3

Measured X-ray photon flux (photons $\mathrm{s}^{-1} \mathrm{~mm}^{-2}$ ) at BL07 of SAGA Light Source downstream of Ge and Si DCM. which is about $10 \times$ bigger than that of the Si DCM. The peak energy is $10 \mathrm{keV}$, which is slightly higher than that of the white SR (Kawamoto et al., 2010) due to the absorption of lowerenergy SR by the Be window and the air between that window and the ion chamber. The $K$ edge of Ge is $11.1 \mathrm{keV}$; therefore, the flux at $12 \mathrm{keV}$ is reduced by about $10 \%$.

Fig. 4 shows the SR spectra as a result of adding different metal filters in the beam path. The spectral data were obtained by $\theta-2 \theta$ scanning of a Si single crystal by an X-ray diffractometer placed on the optical bench of the optical hutch [details of the measurements can be found in a previous report (Yoneyama \& Kawamoto, 2020)]. This result shows that the peak energy can be shifted by changing the metal filter's type and thickness; in particular, it can be set to $15 \mathrm{keV}$ by using $1 \mathrm{~mm}$-thick aluminium and $30 \mathrm{keV}$ by using $0.5 \mathrm{~mm}$-thick copper. Note that we can use all of the SR energy for investigations, whereby the photon flux is about $1000 \times$ higher than that of monochromatic SR.

\subsection{X-ray imager}

Two custom-made microscopic X-ray imagers (Kenvy 1 and 2) and two commercial fiber-coupled $X$-ray imagers with different fields of view are available at the SAGA Light Source. The main specifications of each imager are listed in Table 1.

2.4.1. Microscopic X-ray imagers (Kenvy 1 and Kenvy 2). The microscopic X-ray imagers, Kenvy 1 for white SR

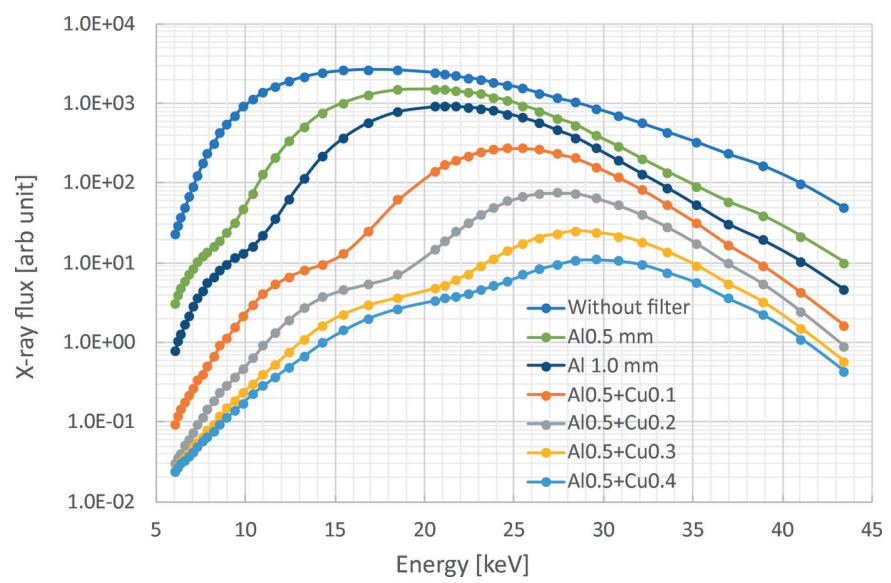

Figure 4

Spectra of quasi-monochromatic SR obtained using various metal filters. Peak energy can be varied by changing the type and thickness of the metal filter. 
Table 2

Main specifications of X-ray imaging methods at SAGA Light Source.

\begin{tabular}{|c|c|c|c|c|c|}
\hline Imaging method & X-ray optics & Scan time & $\begin{array}{l}\text { Spatial } \\
\text { resolution }\end{array}$ & Detector & Application example \\
\hline \multirow[t]{2}{*}{ Micro-CT } & Ge DCM & $1 \mathrm{~h}$ & $2 \mu \mathrm{m}$ & Kenvy 2 & Food, wood, microfossils \\
\hline & Metal filter & $100 \mathrm{~s}$ & $2 \mu \mathrm{m}$ & Kenvy 1 & Wood, microfossils \\
\hline Micro 2D XAFS & Ge DCM & $1 \mathrm{~h}$ & $5 \mu \mathrm{m}$ & Kenvy 2 & $\mathrm{Fe}, \mathrm{Cu}$ powder \\
\hline $\begin{array}{l}\text { Scanning X-ray fluorescence } \\
\text { microscopy (SXFM) }\end{array}$ & - & $1 \mathrm{~h}$ for $200 \mu \mathrm{m} \times 200 \mu \mathrm{m}$ & $1 \mu \mathrm{m}$ & PIN, SDD & $\begin{array}{l}\text { Elemental mapping of seeds, } \\
\text { microfossils and ash }\end{array}$ \\
\hline Grating-based interferometry & Metal filter & $5 \mathrm{~min}$ for $\mathrm{CT}$ & $100 \mu \mathrm{m}$ & Kenvy 1 & Food \\
\hline $\begin{array}{l}\text { Diffraction-enhanced } \\
\text { imaging (DEI) }\end{array}$ & Si DCM & $1 \mathrm{~h}$ for $\mathrm{CT}$ & $30 \mu \mathrm{m}$ & Zyla, VHR & Biomedical and organic samples \\
\hline Crystal-based interferometry & Si DCM & $3 \mathrm{~h}$ for $\mathrm{CT}$ & $30 \mu \mathrm{m}$ & Zyla, VHR & Biomedical and organic samples \\
\hline $\begin{array}{l}\text { Propagation-based } \\
\text { imaging }\end{array}$ & Ge DCM & $1 \mathrm{~h}$ for $\mathrm{CT}$ & $2 \mu \mathrm{m}$ & Kenvy 2 & Food, wood, microfossils \\
\hline Low-temperature CT & Si DCM & $15 \mathrm{~min}$ & $30 \mu \mathrm{m}$ & Zyla, VHR & Food, clathrate hydrates \\
\hline Time-resolved topography & Si DCM & & $30 \mu \mathrm{m}$ & Zyla, VHR & SiC power devices \\
\hline
\end{tabular}

(Yoneyama et al., 2016) and Kenvy 2 for monochromatic SR (Yoneyama et al., 2021), each consist of a phosphor that converts X-rays into visible light, a lens system that transfers the converted visible light to a visible-light camera and an sCMOS visible-light camera (Koch et al., 1998). The lens system employs an infinity optical system, and the magnification can be changed by exchanging the objective lens. Kenvy 1 has a prism in the middle of the lens system; only visible light is reflected $90^{\circ}$ upward to protect the sCMOS camera from being damaged by white SR. The standard phosphor is $\mathrm{Lu}_{3} \mathrm{Al}_{5} \mathrm{O}_{12}: \mathrm{Ce}(\mathrm{LuAG})$ for Kenvy 1 and CsI:Tl (CsI) for Kenvy 2. sCMOS cameras (Andor NEO 5.5 and Zyla 4.2) are used to capture the visible light. The pixel size is $6.5 \mu \mathrm{m}$, while the effective pixel sizes are 1.3 and $0.65 \mu \mathrm{m}$ using $\times 5$ and $\times 10$ objective lenses, respectively.

2.4.2. Fiber-coupled X-ray imagers. The VHR's fiber optics have a taper ratio of $1: 1.5$, the pixel size is $12.5 \mu \mathrm{m}$, the pixel number is $4008 \times 2650$, the field of view is $50 \mathrm{~mm} \times 35 \mathrm{~mm}$, and the image transfer rate is 1.6 frame $\mathrm{s}^{-1}$ for a full image. A $30 \mu$ m-thick $\mathrm{Gd}_{2} \mathrm{O}_{2} \mathrm{~S}: \mathrm{Tb}$ (GOS) phosphor is used to convert the SR into visible light. The Zyla's fiber optics have a taper ratio of $1: 1$, the pixel size is $6.5 \mu \mathrm{m}$, the pixel number is $2560 \times 2180$, the field of view is $16 \mathrm{~mm} \times 13 \mathrm{~mm}$, and the transfer rate is 100 frame s$^{-1}$ for a full image. A $100 \mu \mathrm{m}$-thick CsI phosphor is used.

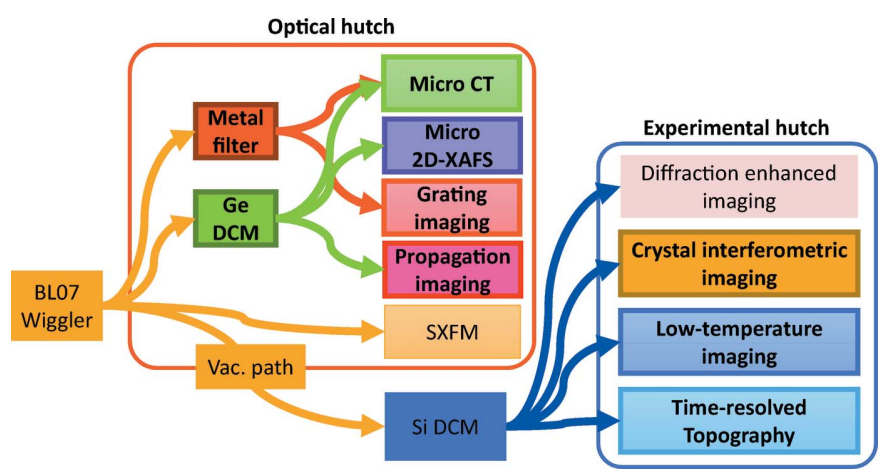

Figure 5

Chart of imaging methods at BL07. Colored arrows indicate the X-ray paths.

\section{Advanced X-ray imaging}

\subsection{Imaging methods}

A chart of the imaging methods available at BL07 is shown in Fig. 5, and the detailed specifications and typical applications of each imaging method are listed in Table 2. Although the SAGA Light Source is a synchrotron facility with a medium ring energy, various imaging methods are possible by combining the white, quasi-monochromatic and monochromatic SR with the various X-ray imagers described above. Details of the imaging methods (indicated by the framed boxes) enabled by the newly installed instruments and exemplary results are described below.

\subsection{Micro-CT}

Fast micro-CT using a combination of Kenvy 1 and quasimonochromatic SR obtained by the metal filter and low-dose micro-CT using a combination of Kenvy 2 and monochromatic SR selected by the Ge DCM (shown as the green boxes in Fig. 5) can be performed in the optical hutch. Both micro-CTs have a parallel-beam geometry in which the propagation distance (PD: the distance between the sample and the X-ray imager) is less than $5 \mathrm{~mm}$ to suppress penumbral blurring. The main advantages are a wide field of view and the ability to use high-flux SR without any intensity loss from X-ray optical devices.

3.2.1. Fast micro-CT using quasi-monochromatic SR. The fast micro-CT system consists of a sample-positioning system and Kenvy 1, as shown in Fig. 6. Both are mounted on a large $Z$ table to adjust the heights relative to the SR optical path simultaneously. The sample-positioning system consists of a large $X$ table and rotational table driven by stepping motors and a small manual $X-Y$ table to adjust the sample center to the rotational center. The sample usually is sealed in a $2 \mathrm{~mm}$ diameter polyethylene tube and attached to the sample holder. The PD is adjusted manually by moving Kenvy 1 along an optical rail.

Fig. 7 shows an observation of a microfossil (benthic foraminifera, Baculogypsina sp.). The exposure time for each image was $100 \mathrm{~ms}$, the number of projections was 2000 over $360^{\circ}$ and the total measurement time was $200 \mathrm{~s} . \mathrm{Cu}(0.1 \mathrm{~mm})$ 


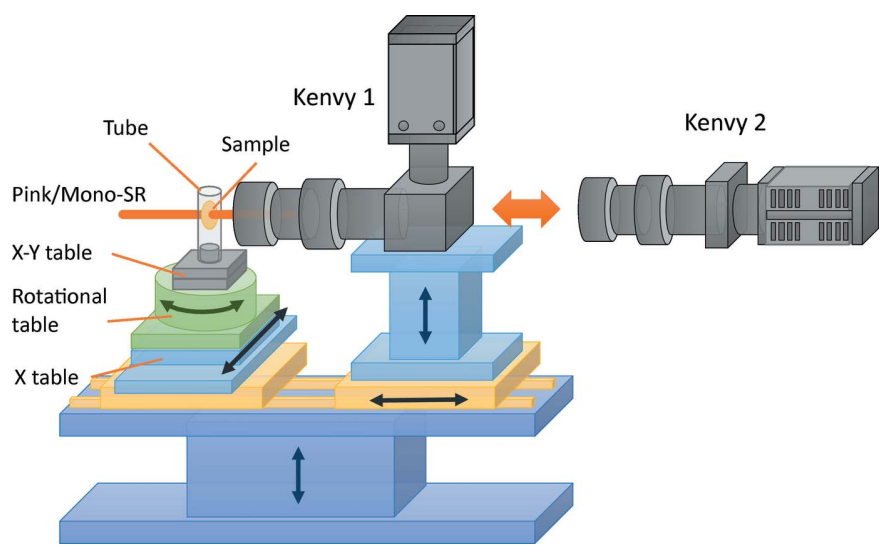

Figure 6

Schematic view of the fast and low-dose micro-CT system. Kenvy 1, for fast micro-CT, and Kenvy 2, for low-dose micro-CT, are exchangeable.

and $\mathrm{Al}(1.5 \mathrm{~mm})$ were used as metal filters, and the peak energy was optimized to $25 \mathrm{keV}$ (see Fig. 4). Owing to the optimized SR energy and good signal-to-noise ratio due to the high intensity of the quasi-monochromatic SR at the selected mean photon energy, not only the surface but also the internal microstructure is depicted with micrometre resolution. The pixel size of the magnified image in the top right and the line profile in the bottom right is $0.65 \mu \mathrm{m}$. As can be seen, fine structures are resolved to 3 pixels; the spatial resolution is estimated to be less than $2 \mu \mathrm{m}(\sim 0.65 \mu \mathrm{m} \times 3)$. The total radiation dose of this observation was calculated to be about 3 MGy.

3.2.2. Low-dose micro-CT using monochromatic SR. Lowdose micro-CT with minimized radiation damage and beam hardening can be performed using the same system, by exchanging Kenvy 1 with Kenvy 2 and the metal filter with the Ge DCM. This micro-CT is suitable for observing samples vulnerable to radiation damage, such as biomedical tissues and organic material. Fig. 8 shows imaging of a Eustoma grand-
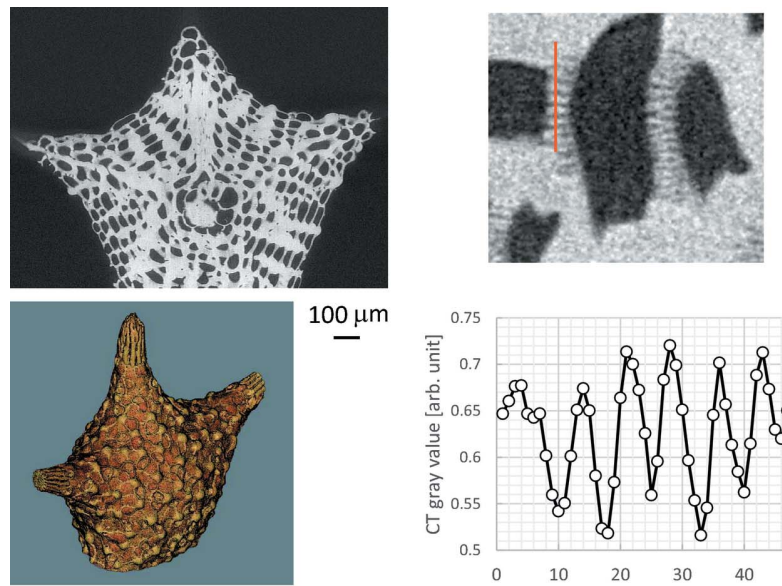

$20 \mu \mathrm{m}$

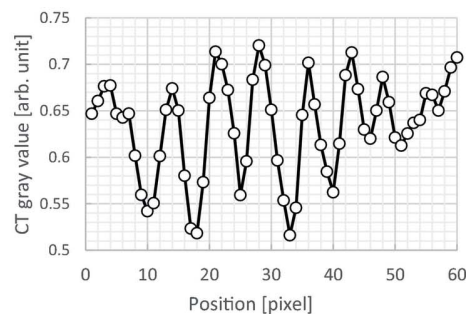

Figure 7

Images of a microfossil. Top left: cross-sectional image; bottom left: 3D volume rendering image; top right: magnified cross-sectional image; bottom right: line profile.
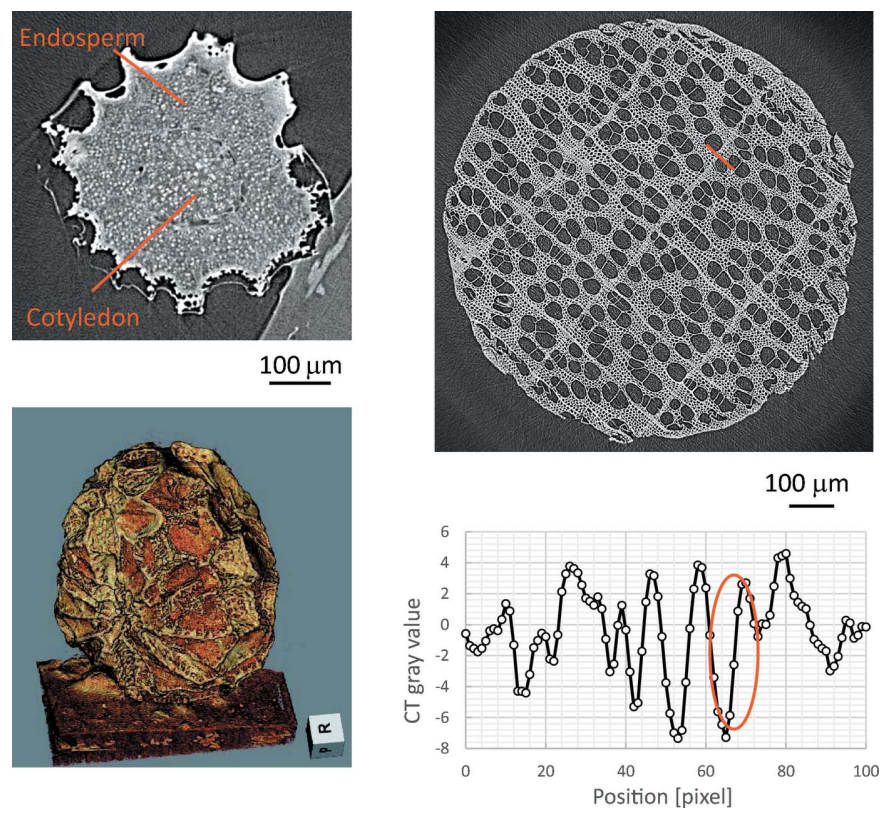

Figure 8

Images of Eustoma grandiflorum seed (top left: cross-sectional image; bottom left: 3D volume rendering image) and white birch (top right: cross-sectional image; bottom right: line profile) from low-dose micro-CT using monochromatic SR.

iflorum seed and white birch. The SR energy was set to $9 \mathrm{keV}$, the exposure time for each image was set to $2 \mathrm{~s}$, and the number of projections was 1000 and 2000 images over $360^{\circ}$ for seed and birch, respectively. The magnification of the objective lens of Kenvy 2 is $\times 5$, so the pixel size is $1.3 \mu \mathrm{m}$.

The results clearly show not only the detailed irregularities in the structure of the seed coat, but also the endosperm and the inside of the cotyledon. The CT gray value of the doublesided tape substrate (paper) is 0.48 , and the fluctuation (standard deviation) of the background region is 0.02 ; therefore, the density resolution is estimated to be $30 \mathrm{mg} \mathrm{cm}^{-3}$, assuming that the paper's density (recycled paper) is $0.80 \mathrm{~g} \mathrm{~cm}^{-3}$. In addition, the CT gray value changes from minimal to maximal within 3 pixels in the orange circle of the line profile (bottom right), so the spatial resolution is estimated to be less than $4 \mu \mathrm{m}(3 \times 1.3 \mu \mathrm{m})$.

3.2.3. Micro-XAFS. Chemical state mappings made from XAFS measurements with micrometre-order spatial resolution (micro-XAFS) can be performed by combining SR monochromated by the Ge DCM with Kenvy 2 (shown as the purple box in Fig. 5). Fig. 9 shows the results of micro-XAFS measurements on a mixture of $\mathrm{Cu}$ and $\mathrm{CuO}_{2}$ powders near the $\mathrm{Cu} K$ edge. Each projection image was obtained by scanning the X-ray energy from 8.89 to $9.08 \mathrm{keV}$ in $0.29 \mathrm{eV}$ increments (12.2 to $11.8^{\circ}$ in $0.0004^{\circ}$ steps of the Ge DCM). The projection images $(130 \mu \mathrm{m} \times 130 \mu \mathrm{m})$ at the top were obtained before the $K$ edge $(8990 \mathrm{eV})$ (left), at the pre-edge $(9000 \mathrm{eV})$ (center) and after the $K$ edge $(9050 \mathrm{eV})$ (right). The spectra at the bottom are logarithms of the average transmitted X-ray intensity at each energy in the blue and orange regions $(14 \times 14$ pixels $)$ in the center image. The exposure time for obtaining each projection image was $2 \mathrm{~s}$ and the total 
measurement time was $30 \mathrm{~min}$. The magnification of the objective lens of Kenvy 2 was $\times 10$, so that the pixel size is $0.65 \mu \mathrm{m}$.

The spectrum in the blue area shows a strong white-line peak characteristic of oxides and a clear pre-edge, indicating that $\mathrm{CuO}_{2}$ is the main component. The orange-area spectrum has the same shape as that of the standard $\mathrm{Cu}$ foil, indicating that $\mathrm{Cu}$ is the main component. The size of each area is $9 \mu \mathrm{m} \times 9 \mu \mathrm{m}(0.65 \mu \mathrm{m} \times 14$ pixels $)$, so the results show that the micro-XAFS system can measure the spatial distribution of chemical states with a spatial resolution of $9 \mu \mathrm{m}$.

\subsection{Phase-contrast $X$-ray imaging (phase imaging)}

Phase-contrast X-ray imaging (phase imaging), which uses the phase shift experienced by X-rays passing through a sample, is a powerful tool for non-destructive 3D observations. Since the cross section of the phase shift for light elements such as carbon, oxygen and nitrogen in the hard X-ray region is more than $1000 \times$ larger than that of absorption (Momose \& Fukuda, 1995; Momose et al., 1996), fine observations can be performed even of biological soft tissues and organic materials, which are mainly composed of light elements. Four kinds of phase imaging for a large field of view can be classified according to the phase-detection method (Bonse \& Busch, 1996; Bravin et al., 2013; Momose, 2020): (i) gratingbased (Talbot) interferometry using X-ray gratings (David et al., 2002; Momose et al., 2003; Weitkamp et al., 2005; Pfeiffer et al., 2006; Yashiro et al., 2010), (ii) crystal-based X-ray interferometry (Momose, 1995; Momose et al., 2001) using a crystal
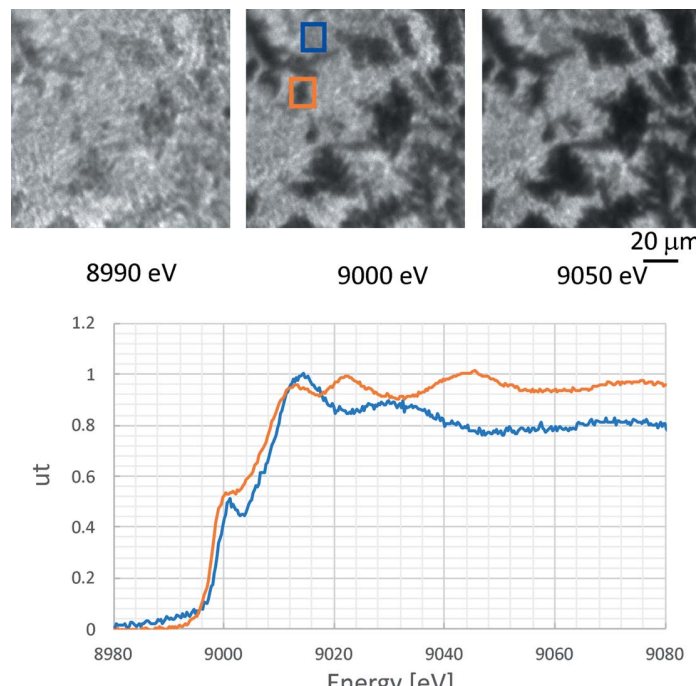

Figure 9

Micro-XAFS measurement results for a mixture of $\mathrm{Cu}$ and $\mathrm{CuO}_{2}$ powders at the $\mathrm{Cu} K$ edge. Top: projection images before $\mathrm{Cu} K$ edge (left), at $\mathrm{Cu}$ pre-edge (center) and after $\mathrm{Cu} K$ edge (right). Bottom: XAFS spectrum of blue and orange area indicated in top-center image.
X-ray interferometer (Bonse \& Hart, 1965), (iii) diffractionenhanced imaging (DEI) using X-ray diffraction of a single crystal (Davis et al., 1995; Chapman et al., 1997) and (iv) propagation-based phase-contrast imaging using Fresnel diffraction (Snigirev et al., 1995; Nugent et al., 1996).

The sensitivity and dynamic range, which indicates the extent to which large differences in density can be observed correctly, of phase imaging are related in a trade-off, and the properties of each method differ significantly, as shown in Table 3. The sensitivity of the crystal-based X-ray interferometry is the highest, and the dynamic ranges of DEI and the propagation-based phase-contrast imaging are the largest (Pagot et al., 2005; Yoneyama et al., 2008; Sun et al., 2013; Yoneyama, Baba, Hyodo et al., 2015). Therefore, selecting an optimized phase-imaging method depending upon the sample density distribution is very important when performing a fine observation, as it takes advantage of the high sensitivity of phase imaging. As shown in Fig. 5, the SAGA Light Source is one of the few SR facilities in the world where all methods are available, i.e. grating-based interferometry for large-field and high-speed phase imaging using quasi-monochromatic SR, DEI and crystal-based X-ray interferometry for large-field and fine phase imaging using monochromatic SR, and propagation-based phase-contrast imaging for high-spatialresolution phase imaging.

3.3.1. Grating-based X-ray interferometry. High-speed phase imaging combined with quasi-monochromatic SR obtained by metal filters and a grating-based X-ray interferometer can be performed in the optical hutch (shown as the orange box in Fig. 5). The imaging system consists of a watercooled metal filter, sample rotational and positioning tables, a phase grating (G1) and its positioning tables, an absorption grating (G2) and its positioning tables, and Kenvy 1, as shown in Fig. 10. The positioning tables of the sample, G1 and G2 are mounted on an optical rail laid on the optical base, and each distance can be easily adjusted by sliding the tables on the rails.

The phase grating (G1) has a period of $4.73 \mu \mathrm{m}$ and is made of Au with a height of $1.9 \mu \mathrm{m}$, which shifts the phase of $20 \mathrm{keV}$ $\mathrm{X}$-rays by $180^{\circ}$; the absorption grating (G2) has a period of $4.8 \mu \mathrm{m}$ and is made of $\mathrm{Au}$ with a height of $150 \mu \mathrm{m}$ to sufficiently absorb X-rays of $20 \mathrm{keV}$ or higher. The calculated Talbot interference distance is $180 \mathrm{~mm}$ for $20 \mathrm{keV}$ X-rays. Each grating was fabricated on the center of a $5^{\prime \prime}(125 \mathrm{~mm}) \mathrm{Si}$ wafer measuring $50 \mathrm{~mm} \times 50 \mathrm{~mm}$, which is sufficiently large 


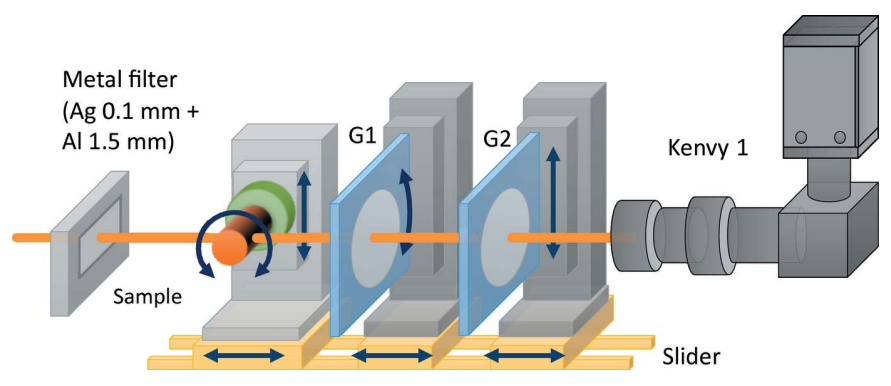

Figure 10

Schematic view of the phase imaging system using an X-ray grating-based interferometer. Quasi-monochromatic SR obtained by a metal filter in the beam path is used for fast phase imaging.

for the beam size $(20 \mathrm{~mm} \times 3 \mathrm{~mm})$. A fringe-scanning method (Bruning et al., 1974), which calculates the phase shift from multiple differential interference images obtained at different phase shifts, is used to detect the quantitative differential phase shifts caused by the sample. To shift the phase, G2 is moved in the $Z$ direction using the positioning table, and the interference images at each phase difference are acquired. Note that the CT measurement is performed using a continuous scanning method (Kibayashi et al., 2012; Yashiro et al., 2018 ) in which G2 is moved in the $Z$ direction, and the sample is simultaneously rotated to shorten the measurement time.

Fig. 11(a) shows 2D absorption-, differential phase- and visibility-contrast images of a calendula seed. The exposure time for each interference image was $0.5 \mathrm{~s}$ and the number of fringe scans was 10 . The metal filters were $1.5 \mathrm{~mm}$-thick $\mathrm{Al}$ and $0.5 \mathrm{~mm}$-thick $\mathrm{Ag}$, so the peak energy was $22 \mathrm{keV}$. The magnification of the object lens of Kenvy 1 is 2 , the field of view is $8.3 \mathrm{~mm} \times 7.0 \mathrm{~mm}$, and the pixel size is $3.2 \mu \mathrm{m}^{2}$. The numerical aperture of the $\times 2$ lens is very small (0.055), so a long exposure time was required. These results show that

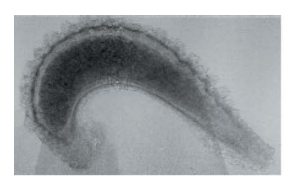

Absorption

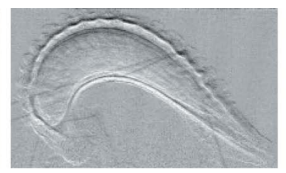

Differential phase

(a)

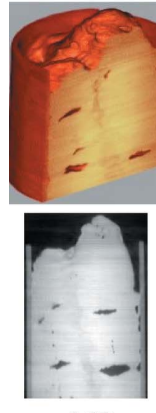

$0: 00$

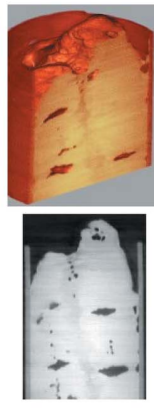

$0: 30$

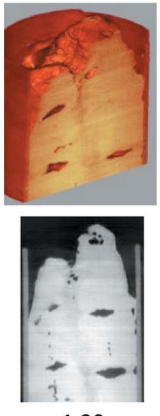

$1: 30$

(b)

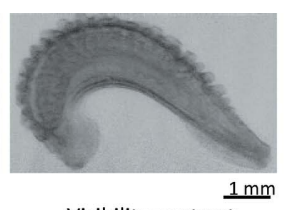

Visibility contrast

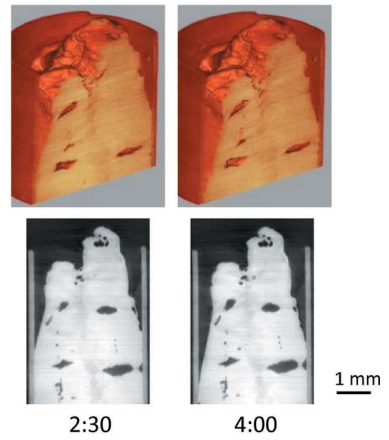

2:30

4:00

Figure 11

(a) 2D images of calendula seed and (b) time-resolved 3D images (upper) and sectional images (lower) of cooked rice over $4 \mathrm{~h}$ by grating-based interferometry. the contrasts of each image differ from one another. The absorption-contrast image shows the dense internal regions and the visibility-contrast image shows the detailed internal structure, while the differential phase-contrast image shows the fine surface structure.

Fig. 11(b) shows the time-resolved 3D phase-contrast images of cooked rice that were acquired over the course of $4 \mathrm{~h}$. The exposure time of each interference image was $0.1 \mathrm{~s}$, the number of fringe scans was 5 , the number of projections was 200 and the total measurement time was $100 \mathrm{~s}$ because continuous scanning was used. The images show that the rice dries out and gradually shrinks while the bubbles inside gradually increase in size. The density decreased by about $20 \%$ during the measurement.

3.3.2. Crystal-based X-ray interferometry. Crystal-based $\mathrm{X}$-ray interferometry detects phase shifts using a crystal X-ray interferometer (XI) cut from a large perfect-crystal ingot. The conventional monolithic triple Laue case (LLL) XI (called the Bonse-Hart type) has three parallel crystal wafers, as shown in Fig. 12(a), and it functions in the same way as a MachZehnder interferometer in the visible-light region. In particular, incident X-rays are split by the first wafer (splitter, S), reflected by the second wafer (mirror, $\mathrm{M}$ ) and combined at the third wafer (analyzer, A) to form two interference X-ray beams. The phase shift caused by the sample placed in the object beam path is converted into intensity changes in the interference beams due to wave superposition, which are detected directly; hence, the sensitivity of this method is the highest among the phase imaging methods.

A phase-contrast X-ray imaging system equipped with a two-crystal XI consisting of two crystal blocks, each having two crystal wafers, has been developed for non-destructive 3D observation of large samples at the Photon Factory in Japan (Yoneyama et al., 2013), and researchers have taken advantage of its high sensitivity to carry out various observations in biomedicine (Yoneyama et al., 2006; Noda-Saita et al., 2006; Takeda et al., 2012; Yamada et al., 2012; Kishimoto et al., 2016; Thet Thet et al., 2018; Kanahashi et al., 2019), environmental science (Takeya, 2006) and industrial science (Takeya et al., 2012; Mimachi et al., 2015; Takamatsu et al., 2018, 2020). The advantages of two-crystal XI are its large field of view $\left(>30 \mathrm{~mm}^{2}\right)$ and large sample space; on the other hand, operation of such an interferometer requires extremely high

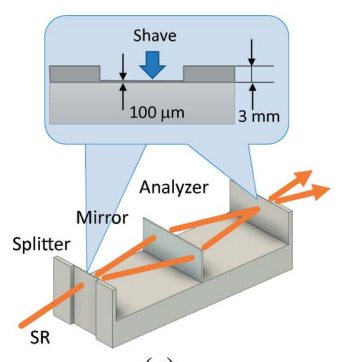

(a)

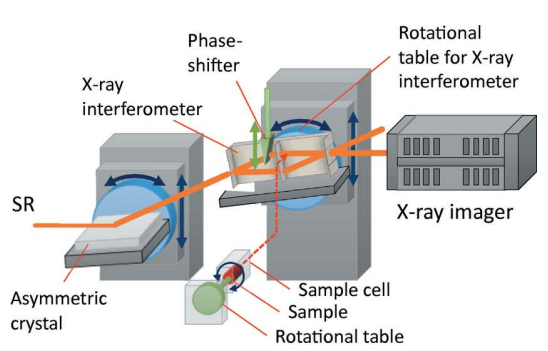

(b)
Figure 12

(a) Schematic views of the monolithic XI with thin splitter and analyzer and $(b)$ imaging system using XI. 
rotational stability (sub-nrad order) between the crystal blocks (Becker \& Bonse, 1974). Since the beam size of BL07 is small $(20 \mathrm{~mm} \times 20 \mathrm{~mm})$, we installed a monolithic XI that uses Si (220) diffraction (Sharan Instruments, Japan). The absorption of one $1 \mathrm{~mm}$-thick crystal wafer is about $25 \%$ for $17.8 \mathrm{keV} \mathrm{SR}$, and the intensity of the interference beams is decreased to less than $5 \%$ of the incident SR intensity. To reduce the wafer's absorption, the thicknesses of the splitter (S) and analyzer (A) have been reduced from 3000 to $100 \mu \mathrm{m}$ by shaving, as shown in Fig. 12(a). The coherence length of the $\mathrm{SR}$ is a few micrometres, and the differences in the spacing between the crystal wafers ( $\mathrm{S}-\mathrm{M}$ and $\mathrm{M}-\mathrm{A}$ ) have to be kept equal or less for good interference; thus, the incident side of $S$ and outgoing side of $\mathrm{A}$ are shaved. Note that the thickness of $\mathrm{M}$ is $1 \mathrm{~mm}$.

Fig. 12(b) shows a schematic view of the phase-contrast imaging system using XI with thinned wafers (shown as the yellow box in Fig. 5). The system consists of an asymmetric crystal (to expand the SR vertically), the XI and its positioning system, the sample holder and its positioning system, and the X-ray imager (Zyla or VHR). The height of the incident monochromatic SR is less than $5 \mathrm{~mm}$, even at experimental hutch 2 , so we installed an asymmetric crystal with an asymmetry angle of $5^{\circ}$ to expand the beam in the vertical direction. One of the interference beams generated by the XI is detected by the X-ray imager. Since both the asymmetric crystal and the interferometer require sub- $\mathrm{rad}$ angular stability to perform fine observations, they are mounted on high-precision goniometers to precisely control the incidence angle. The entire imaging system is covered with a curtain to shield the airflow in the experimental hutch, and the temperature fluctuation around the system is no more than $0.5^{\circ} \mathrm{C}$ during the course of $12 \mathrm{~h}$. Note that the monolithic X-ray interferometer is affected by sample heat because the distance between the sample and the crystal wafer is short. Therefore, observations are limited to samples at the same temperature level as room temperature.

If the spacing of the interference fringes is narrower than the spatial resolution of the X-ray imager, the fringes cannot be detected accurately, and phase unwrapping cannot be performed correctly [phase-unwrapping error is the inability to detect whether the phase jump is $2 \pi n$ or $2 \pi(n+1)]$. To eliminate this error, the sample is placed in a liquid such as water, saline solution or ethylene glycol, whichever has the closest density to that of the sample, in order to widen the fringe spacing at the sample's boundary. In the CT measurement depicted in Fig. 12(b), the sample is rotated around the horizontal axis in the liquid cell. As with grating-based interferometry, the fringe scanning method is used for quantitatively measuring phase shifts; therefore, an acrylic wedge is inserted vertically into the interferometer's optical path as a phase shifter, and the phase is shifted by moving the wedge up and down.

Fabrication of a monolithic XI with the A-wafer center thinned by etching and an interference image of a plastic ball without blurring was reported (Hirano \& Momose, 1999). However, no operational result of XI with thinned S and A

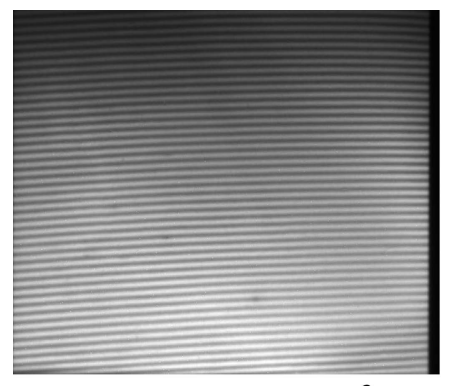

(a)

$\underline{2 \mathrm{~mm}}$

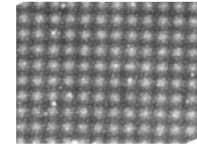

(b)

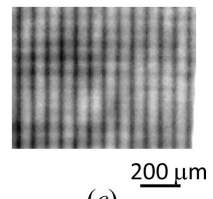

(c)
Figure 13

(a) X-ray interference image acquired by the XI with thin S and A wafers. Interference images of Au mesh (400 line-space inch $^{-1}$ ) acquired by XI with thinned wafers $(b)$ and conventional XI ( $1 \mathrm{~mm}$-thick wafers) (c). Image $(c)$ is blurred in the diffraction direction (vertical direction) by the Borrmann fan effect.

wafers has been reported in the literature, so we first tested the performance (intensity, uniformity and visibility) of the interference pattern using monochromatic $17.8 \mathrm{keV}$ SR. Fig. 13(a) shows an X-ray interference pattern obtained using the Zyla with a $1 \mathrm{~s}$ exposure time. The size of the pattern (field of view) is about $15 \mathrm{~mm} \times 12 \mathrm{~mm}$, which is large enough to observe a small sample of animal organs. The spatial distribution of visibility is almost uniform; average visibility is $40 \%$, which is lower than that of a conventional monolithic XI $(\sim 80 \%)$. Note that the intensity at the top of the pattern is lower because of the lower incident SR intensity.

The advantage of the XI with thinned S and A wafers is not only the SR's high throughput but also its lack of image blur from the Borrmann fan effect. According to the dynamical theory of X-ray diffraction (Batterman \& Cole, 1964), the $\mathrm{X}$-ray beam path inside the crystal wafer is very sensitive to deviations from the Bragg angle, and even a small angular change causes a large positional shift of the outgoing beam. Any slight angular change caused by sample refraction changes the beam path in the A wafer significantly, resulting in a blurred interference image (the Borrmann fan effect). Thus, the XI with a thin A wafer makes it possible to perform high-spatial-resolution observations. Figs. 13(b) and 13(c) compare the interference patterns of an Au mesh (400 linespace inch ${ }^{-1}$ ) obtained using the XI with thinned $\mathrm{S}$ and $\mathrm{A}$ wafers and a conventional XI ( $1 \mathrm{~mm}$-thick wafers). The patterns were recorded under the same conditions, i.e. X-ray energy of $17.8 \mathrm{keV}$ and Zyla imager, except for $(c)$, which was obtained at the beamline BL16B2 of SPring-8. The mesh is clearly visible in the vertical and horizontal directions in $(b)$; in contrast, the mesh is blurred in the vertical direction (parallel to the diffraction plane) in $(c)$. The blurring caused by the Borrmann fan effect is given approximately by the product of the crystal thickness and the Bragg angle, and it is calculated to be $35 \mu \mathrm{m}$ for the $100 \mu \mathrm{m}$ wafer and $350 \mu \mathrm{m}$ for the $1 \mathrm{~mm}$ wafer. Therefore, the blurring in $(c)$ is mainly caused by the Borrmann fan effect.

Fig. 14(a) shows the phase maps (spatial distributions of phase shift) of a mouse kidney obtained using the XI with thinned S and A wafers. The SR energy was $17.8 \mathrm{keV}$, the exposure time for acquiring each interference image was $10 \mathrm{~s}$ 


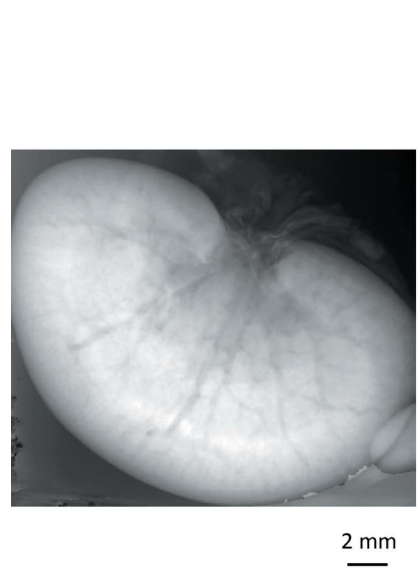

(a)
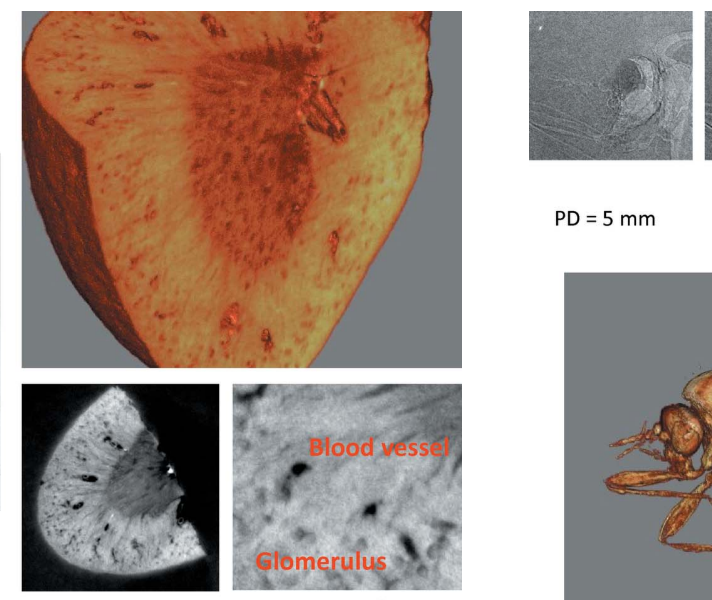

$2 \underline{\mathrm{mm}}$

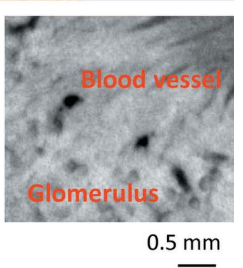

(b)

$\mathrm{PD}=5 \mathrm{~mm}$
Figure 14

(a) Phase map and (b) 3D image (top), whole sectional image (bottom left) and magnified sectional image (bottom right) of mouse kidney by crystal-based X-ray interferometry.

and a six-step fringe scanning method was used. The Zyla was used as the X-ray imager, so the pixel size is $6.5 \mu \mathrm{m}$. Owing to the high sensitivity of the crystal-based X-ray interferometry, the microvasculature inside the kidney could be visualized in detail without using any contrast agent.

Fig. 14(b) shows the 3D and sectional images of a piece of mouse kidney obtained by phase-contrast CT. The X-ray energy was $19.0 \mathrm{keV}$, the exposure time for one interference image was $5 \mathrm{~s}$ and the phase map of each projection angle was acquired using a three-step fringe scanning method. The number of projections was 500 over $360^{\circ}$ and the total measurement time was about $3 \mathrm{~h}$. Detailed structures of blood vessels, the cortex and glomeruli inside the kidney are clearly visible. The density resolution calculated from the standard deviation in the background area is $1.0 \mathrm{mg} \mathrm{cm}^{-3}$, which is about two times larger than that of the phase imaging system at the Photon Factory (Yoneyama et al., 2013). The deterioration in resolution may be due to the low SR intensity and the low visibility of the interference image. A blood vessel with a diameter of $30 \mu \mathrm{m}$ is visualized in the enlarged image (bottom right); the spatial resolution is estimated to be finer than $30 \mu \mathrm{m}$.

3.3.3. Propagation-based phase-contrast imaging. The propagation-based phase-contrast imaging detects phase shifts by using Fresnel diffraction and does not require any additional X-ray optical components. Therefore, the method is suitable for micro-phase-contrast CT and can be performed by combining SR monochromated using the Ge DCM and Kenvy 2 (shown as the red box in Fig. 5). The instrumentation configuration is the same as the one used to make Fig. 6, except that Kenvy 2 is separated from the sample. Fig. 15(a) shows projection images acquired at different PDs of an insect (Ceratopogonidae) trapped in amber. The edges of the insect become more pronounced and the detailed structures can be visualized by increasing the PD. On the other hand, the image becomes blurred when the PD exceeds $50 \mathrm{~mm}$.
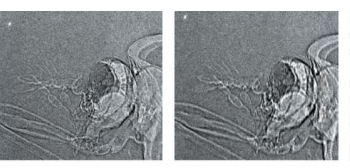
$25 \mathrm{~mm}$

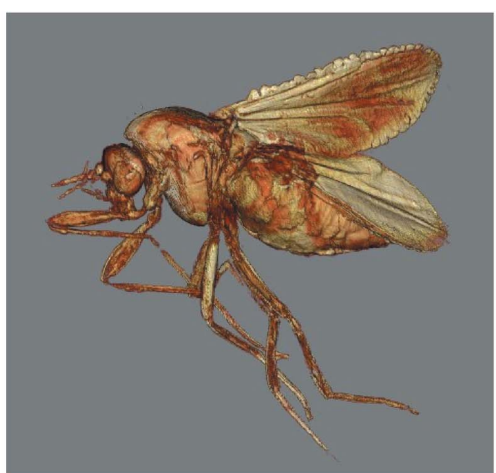

(b)
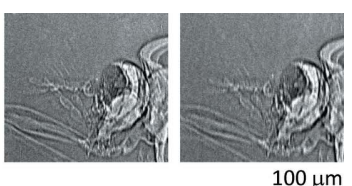

$75 \mathrm{~mm}$

$100 \mathrm{~mm}$

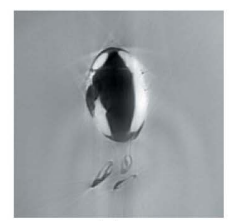

(c)

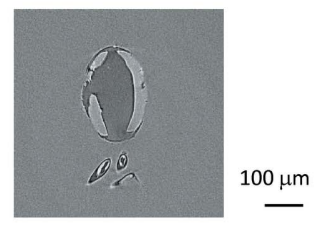

(d)
Figure 15

(a) Projection image of an insect (Ceratopogonidae) in amber obtained by propagation-based phase-contrast imaging. Edges are enhanced by increasing the PD. (b) 3D image and (c) cross-sectional image near the abdomen of an insect (Ceratopogonidae) in amber. $\mathrm{S} / \mathrm{N}$ is $10 \times$ higher than that of an absorption-contrast image $(d)$ of the same slice.

Figs. 15(b) and 15(c) show a 3D image and a cross-sectional image near the abdomen of the same sample. The sectional image was reconstructed by conventional filtered backprojection after phase retrieval using an algorithm for a single distance (Weitkamp et al., 2011). Each projection image was acquired at a PD of $20 \mathrm{~mm}$ and $10 \mathrm{keV} \mathrm{SR}$. The exposure time was $2 \mathrm{~s}$, and the number of projections was 1000 over $360^{\circ}$. The images clearly illustrate the detailed structures inside the insect's body and its wings. Fig. 15(d) shows a sectional image reconstructed by conventional filtered back-projection without phase retrieval for the same CT data (absorptioncontrast image). The signal-to-noise ratio $(\mathrm{S} / \mathrm{N})$ computed using the CT value in the abdomen as the signal and fluctuations (standard deviation) in the background region as noise in $(c)$ is about $10 \times$ higher than that in $(c)$. Note that $(d)$ was measured at a single distance, so the density is not quantifiable as the precise values for the index of refraction are unknown.

\subsection{Low-temperature CT}

3D observations made at low temperature of samples such as clathrate hydrates and frozen foods can be performed using a combination of SR monochromated by the Si DCM and a sample-cooling (cryogenic) system in experimental hutch 2, as shown in Fig. 16 (shown as the blue box in Fig. 5). The custom-made cryogenic system consists of a liquid-nitrogen evaporator, a low-temperature dry-nitrogen-gas blowing nozzle $15 \mathrm{~mm}$ in diameter, a liquid-nitrogen tank and a controller. The sample is cooled by flowing evaporated dry nitrogen gas at $123 \mathrm{~K}$ from a nozzle. To prevent condensation of ice on the sample, the gas flow is exhausted using an aspirator from the sample's opposite side. The sample posi- 


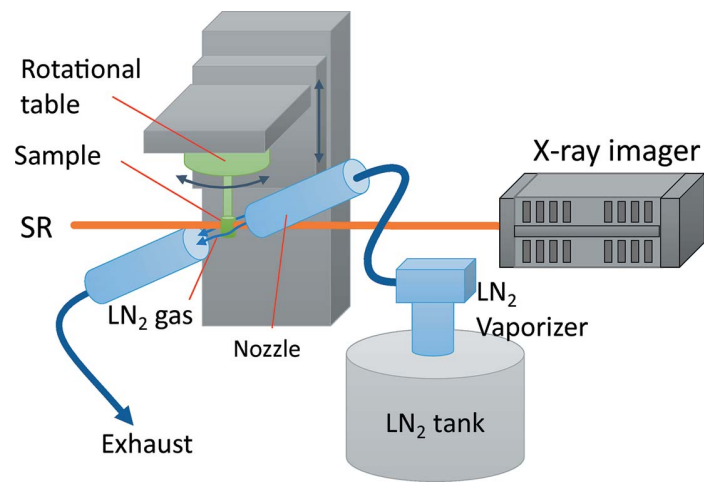

Figure 16

Schematic view of the low-temperature CT system. The sample is kept at low temperature by nitrogen gas flow.

tioning system for the CT measurements consists of remotely controlled rotation and vertical tables.

Fig. 17 shows a 3D image (left), whole sectional image (center) and magnified sectional image (right) of ice cream confined in a polypropylene tube with a $2 \mathrm{~mm}$ diameter. The $\mathrm{X}$-ray energy was $15 \mathrm{keV}$, the exposure time was $1 \mathrm{~s}$, the number of projections was 1000 over $360^{\circ}$ and the total measurement time was $1000 \mathrm{~s}$. The Zyla was used as the X-ray imager, so the pixel size is $6.5 \mu \mathrm{m}$. Low-temperature nitrogen gas was blown continuously over the sample to keep it cold during the measurement. The magnified sectional image reveals air bubbles and ice, as was found in previous reports (Pinzer et al., 2012; Dalen et al., 2016). The density resolution is estimated to be $0.10 \mathrm{~g} \mathrm{~cm}^{-3}$ using the standard deviation of the background region and the polypropylene tube's CT value. The spatial resolution is higher than $20 \mu \mathrm{m}$, because the images show air bubbles that are about $20 \mu \mathrm{m}$ in diameter.

\subsection{Time-resolved topography}

$\mathrm{X}$-ray topography is a high-sensitivity method for detecting dislocations and defects in crystals. It is widely used to evaluate crystalline materials such as silicon, silicon carbide $(\mathrm{SiC})$, diamond and gallium nitride. Since the size of a typical dislocation, such as a screw dislocation, is very small (a few micrometres) compared with a crystal wafer or semiconductor device, a detecting device with high spatial resolution and a
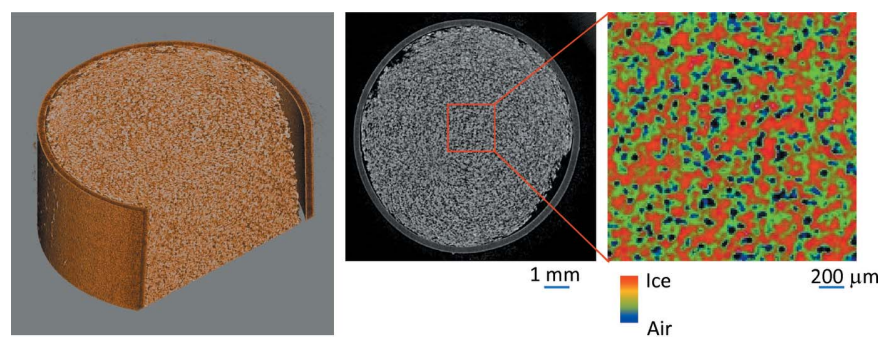

Figure 17

3D (left), whole sectional (center) and magnified sectional (right) images of ice cream observed at low temperature. Air bubbles and ice pieces are clearly visualized. large field of view is indispensable. X-ray films or nuclear emulsion detectors can be used for this purpose, but their measurements are limited to static observations. It has been revealed that stacking faults expand in $\mathrm{SiC}$ power devices when they are in operation, which may cause severe deterioration of the device's electrical characteristics. Furthermore, stacking faults are much larger than screw dislocations; thus, they can be detected dynamically with an X-ray imager such as the Zyla. We have developed a time-resolved X-ray topography system to observe the expansion of stacking faults inside operating semiconductor devices by using a combination of SR monochromated using the Si DCM and the Zyla (shown as the cyan box in Fig. 5). Note that the white SR at BL07 is very strong, and its use might have accelerated the expansion of stacking faults and dislocations; thus, monochromatic SR was used.

Fig. 18(a) is a schematic view of the topography system, which mainly consists of an X-ray diffractometer with a $2 \theta$ arm, a sample positioner, an X-ray imager (Zyla), a samplecooling system and a power supply for operating the device being tested. The monochromatic SR selected by the Si DCM is formed by a four-blade slit (not shown) and irradiates the sample. The X-rays diffracted by the sample are detected by the Zyla attached to the $2 \theta \mathrm{arm}$. The device is mounted directly on the bonded copper substrate by soldering and set on a sample holder ( $\mathrm{Cu}$ block), which is water-cooled to $12^{\circ} \mathrm{C}$ using a high-precision chiller.

We also developed a feedback system to stabilize the $\mathrm{X}$-ray's incident angle to the sample. The system consists of a tilt table driven by a piezoelectric translator (PZT), a PZT controller, digital-analog data acquisition (DAQ) and a control PC, as shown in Fig. 18(b). If the incident angle drifts because of the temperature change caused by operation of the device, the intensities on the left or right sides of the topographic image increase, as shown in Fig. 18(b). The feedback system detects the intensity difference, calculates the compensation voltage of the PZT needed to make the intensity the same, and changes the voltage applied to the PZT via the DAQ to correct the incident angle.

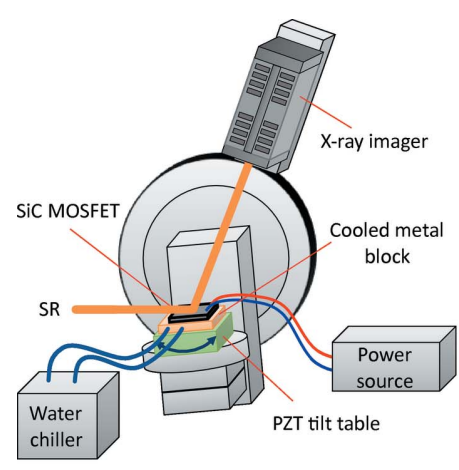

(a)

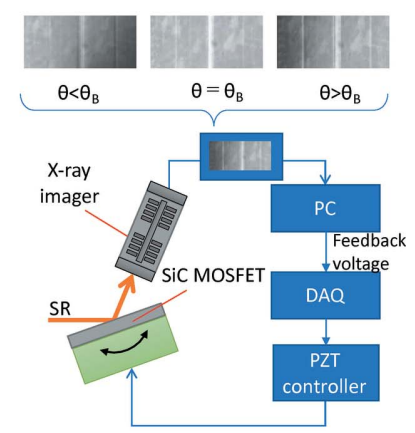

(b)
Figure 18

(a) Schematic view of the time-resolved topography system and (b) feedback system for stabilizing the X-ray's incident angle using observed topography. 
The tilt table's angular range is $\pm 0.15^{\circ}$, which is sufficiently larger than the angular drift caused by the temperature change $\left(\sim 100^{\circ} \mathrm{C}\right)$. The positioning accuracy (angular resolution) of the table is less than $1 / 100 \mu \mathrm{rad}$, and the X-ray's incident angle can be controlled with sufficient accuracy to satisfy the Bragg diffraction condition of the $\mathrm{SiC}$. The exposure time needed for obtaining a topographic image is usually $1-10 \mathrm{~s}$, so the operating frequency of the feedback is less than $1 \mathrm{~Hz}$; however, the thermal drift rotation is very slow, from several minutes to several hours, so that it can be suppressed sufficiently even at a slow operating frequency.

Time-resolved topography of a $\mathrm{SiC}$ metal-oxide-semiconductor field-effect transistor (MOSFET) was performed using monochromatic $10 \mathrm{keV} \mathrm{SR}$. SiC $(0-2210)$ diffraction was used for the observation because the stacking faults in the SiC MOSFET appear as bands (Konishi et al., 2021). The calculated incidence and diffraction angles were $17.4^{\circ}$ and $83.6^{\circ}$ for $4 \mathrm{H}-\mathrm{SiC}$ with $4^{\circ}$ off-axis (0001). Fig. $19(a)$ shows timeresolved topographic images of the device in operation. The exposure time was $10 \mathrm{~s}$, but the interval of each displayed image was 2 min because of its slow expansion rate. The growth of a stacking fault over the course of $20 \mathrm{~min}$ is clearly visible. Fig. 19(b) shows time-resolved topographic images of the same device taken with a $25 \mathrm{~s}$ interval. The expansion was faster than that of the stacking fault in the upper image and saturated within $2 \mathrm{~min}$. Also, it rapidly extended downwards after saturation. Fig. 19(c) shows a time chart of the diffracted $\mathrm{X}$-ray intensity of the orange line in the topography. The chart indicates that the origin of the stacking fault was not one point but rather several points and that the faults spread gradually before finally merging. These results show that time-resolved topography makes it possible to quantitatively evaluate how stacking faults expand in time and at what speed.

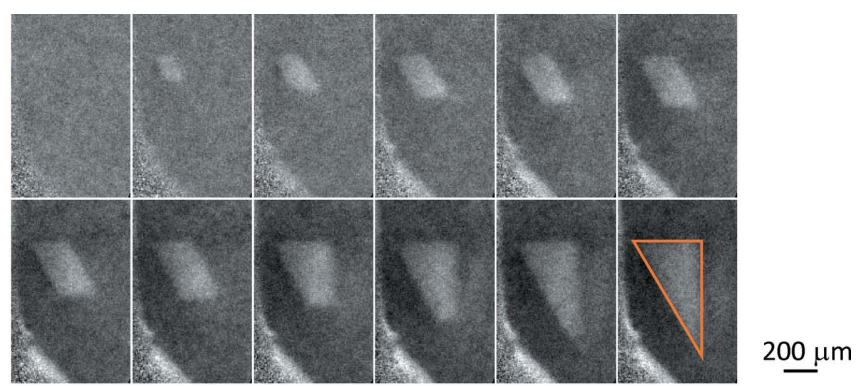

(a)

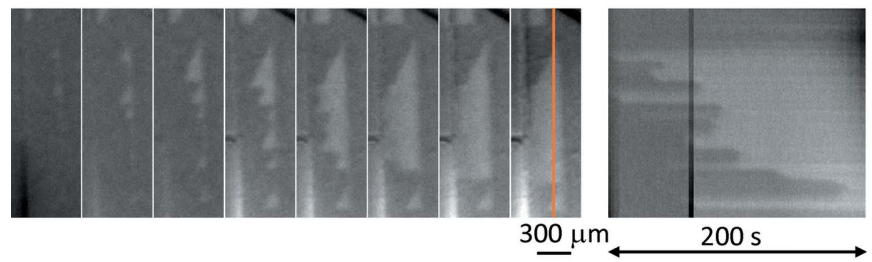

(b)

(c)

Figure 19

(a) Topographic images of a slowly expanding stacking fault (taken at 2 min intervals) and (b) a quickly expanding stacking fault (taken at $25 \mathrm{~s}$ intervals), and (c) time chart of diffracted X-ray intensity of the orange line in $(b)$.

\section{Conclusion}

We have been developing various advanced imaging methods and systems for non-destructive observations of various samples so that researchers in fields ranging from biomedicine to functional materials can take advantage of the brilliant SR emitted from the superconducting wigglers at BL07 of the SAGA Light Source. By combining white, quasi-monochromatic and monochromatic SR, fast and low-dose 3D microCT observations and 2D micro-XAFS observations can be performed at the optical hutch. Additionally, numerous largearea phase-contrast X-ray imaging techniques are available, including grating-based X-ray interferometry, DEI, crystalbased X-ray interferometry and propagation-based phasecontrast imaging, can be used to perform high-density-resolution observations of biomedical and organic material samples. Low-temperature samples, such as of frozen foods, can be observed with the low-temperature CT system. Moreover, the time-resolved topography system can be used to analyze quantitatively, e.g. the expansion of stacking faults in power devices in operation. The next step in our developments will be to devise and install high-speed SXFM using optimized focusing mirrors (Matsuyama et al., 2016), highspeed CT combined with a fast X-ray imager and white SR, and a micro-phase-contrast low-temperature $\mathrm{CT}$ for biomedical and industrial applications.

\section{Acknowledgements}

We would like to express our appreciation to Mr Kenichirou Sakamoto and Ms Akane Matsumoto of the Agricultural Research Center of Saga Prefecture for preparing the Eustoma grandiflorum seeds. We thank Professor Wataru Yashiro of Tohoku University for valuable communications on phase-contrast imaging.

\section{Funding information}

The study was carried out under proposal Nos. 180202I, 190202I and 200202I of the Committee of the SAGA Light Source and proposal Nos. 2019A5400 and 2019B5400 of SPring-8. The biological experiment was approved by the President of Kitasato University through the judgment of the Animal Care and Use Committee of Kitasato University (approval No. 14-02).

\section{References}

Arai, S., Yonezawa, Y., Okazaki, N., Matsumoto, F., Shibazaki, C., Shimizu, R., Yamada, M., Adachi, M., Tamada, T., Kawamoto, M., Tokunaga, H., Ishibashi, M., Blaber, M., Tokunaga, M. \& Kuroki, R. (2015). Acta Cryst. D71, 541-554.

Batterman, B. W. \& Cole, H. (1964). Rev. Mod. Phys. 36, 681-717. Becker, P. \& Bonse, U. (1974). J. Appl. Cryst. 7, 593-598.

Bonse, U. \& Busch, F. (1996). Prog. Biophys. Mol. Biol. 65, 133-169.

Bonse, U. \& Hart, M. (1965). Appl. Phys. Lett. 6, 155-156.

Bravin, A., Coan, P. \& Suortti, P. (2013). Phys. Med. Biol. 58, R1-R35. Bruning, J. H., Herriott, D. R., Gallagher, J. E., Rosenfeld, D. P., White, A. D. \& Brangaccio, D. J. (1974). Appl. Opt. 13, 2693-2703. 
Chapman, D., Thomlinson, W., Johnston, R. E., Washburn, D., Pisano, E., Gmür, N., Zhong, Z., Menk, R., Arfelli, F. \& Sayers, D. (1997). Phys. Med. Biol. 42, 2015-2025.

Dalen, G., v, Bent, J., Guo, E., Zeng, G., Rockett, P. \& Lee, P. D. (2016). Conference: Bruker Micro-CT User Meeting, Luxembourg, pp. 1-9. Bruker.

David, C., Nöhammer, B., Solak, H. H. \& Ziegler, E. (2002). Appl. Phys. Lett. 81, 3287-3289.

Davis, T. J., Gao, D., Gureyev, T. E., Stevenson, A. W. \& Wilkins, S. W. (1995). Nature, 373, 595-598.

Hirano, K. \& Momose, A. (1999). Jpn. J. Appl. Phys. 38, L1556L1558.

Ishiji, K., Kawado, S. \& Hirai, Y. (2011). Phys. Status Solidi A, 208, 2516-2521.

Kanahashi, T., Yamada, S., Yoneyama, A. \& Takakuwa, T. (2019). Anat. Rec. 302, 1968-1976.

Kawamoto, M., Sumitani, K., Okajima, T., Garrett, R., Gentle, I., Nugent, K. \& Wilkins, S. (2010). AIP Conf. Proc. 1234, 355-358.

Kibayashi, S., Harasse, S., Yashiro, W. \& Momose, A. (2012). AIP Conf. Proc. 1466, 261-265.

Kishimoto, H., Yamada, S., Kanahashi, T., Yoneyama, A., Imai, H., Matsuda, T., Takeda, T., Kawai, K. \& Suzuki, S. (2016). Dev. Dyn. 245, 123-131.

Koch, A., Raven, C., Spanne, P. \& Snigirev, A. (1998). J. Opt. Soc. Am. A, 15, 1940-1951.

Konishi, K., Fujita, R., Kobayashi, K., Yoneyama, A., Ishiji, K., Okino, H., Shima, A. \& Ujihara, T. (2021). J. Appl. Phys. 130, 145703.

Matsuyama, S., Nakamori, H., Goto, T., Kimura, T., Khakurel, K. P., Kohmura, Y., Sano, Y., Yabashi, M., Ishikawa, T., Nishino, Y. \& Yamauchi, K. (2016). Sci. Rep. 6, 24801.

Mimachi, H., Takahashi, M., Takeya, S., Gotoh, Y., Yoneyama, A., Hyodo, K., Takeda, T. \& Murayama, T. (2015). Energy Fuels, 29, 4827-4834.

Momose, A. (1995). Nucl. Instrum. Methods Phys. Res. A, 352, 622628.

Momose, A. (2020). Phys. Med. 79, 93-102.

Momose, A. \& Fukuda, J. (1995). Med. Phys. 22, 375-379.

Momose, A., Kawamoto, S., Koyama, I., Hamaishi, Y., Takai, K. \& Suzuki, Y. (2003). Jpn. J. Appl. Phys. 42, L866-L868.

Momose, A., Takeda, T., Itai, Y. \& Hirano, K. (1996). Nat. Med. 2, 473-475.

Momose, A., Takeda, T., Yoneyama, A., Koyama, I. \& Itai, Y. (2001). Nucl. Instrum. Methods Phys. Res. A, 467-468, 917-920.

Noda-Saita, K., Yoneyama, A., Shitaka, Y., Hirai, Y., Terai, K., Wu, J., Takeda, T., Hyodo, K., Osakabe, N., Yamaguchi, T. \& Okada, M. (2006). Neuroscience, 138, 1205-1213.

Nugent, K. A., Gureyev, T. E., Cookson, D. F., Paganin, D. \& Barnea, Z. (1996). Phys. Rev. Lett. 77, 2961-2964.

Ohtani, R., Tokita, T., Takaya, T., Iwata, K., Kinoshita, M., Matsumori, N., Nakamura, M., Lindoy, L. F. \& Hayami, S. (2017). Chem. Commun. 53, 13249-13252.

Okajima, T., Jia, J. \& Shigesato, Y. (2018). Mater. Res. Express, 5, 046412.

Pagot, E., Fiedler, S., Cloetens, P., Bravin, A., Coan, P., Fezzaa, K., Baruchel, J., Härtwig, J., von Smitten, K., Leidenius, M., Karjalainen-Lindsberg, M. L. \& Keyriläinen, J. (2005). Phys. Med. Biol. 50, 709-724.

Pfeiffer, F., Weitkamp, T., Bunk, O. \& David, C. (2006). Nat. Phys. 2 , 258-261.
Pinzer, B. R., Medebach, A., Limbach, H. J., Dubois, C., Stampanoni, M. \& Schneebeli, M. (2012). Soft Matter, 8, 4584-4594.

Ravel, B. \& Newville, M. (2005). J. Synchrotron Rad. 12, 537-541.

Sakamoto, K., Nishi, M., Ishiji, K., Takatori, Y. \& Chiwata, R. (2019). XIII International Symposium on Flower Bulbs and Herbaceous Perennials, pp. 73-78. International Society for Horticultural Science (ISHS).

Snigirev, A., Snigireva, I., Kohn, V., Kuznetsov, S. \& Schelokov, I. (1995). Rev. Sci. Instrum. 66, 5486-5492.

Sumitani, K., Ishiji, K., Kawamoto, M., Yoneyama, A., Tabata, M., Okajima, T. \& Hirai, Y. (2013). J. Phys. Conf. Ser. 425, 192013.

Sumitani, K., Magome, E. \& Hirai, Y. (2011). IOP Conf. Ser.: Mater. Sci. Eng. 24, 012018.

Sun, J., Liu, P., Irvine, S., Pinzer, B., Stampanoni, M. \& Xu, L. X. (2013). J. Instrum. 8, C06003.

Tabata, M. \& Ueda, S. (2017). 7th International Symposium on Indigenous Knowledge, pp. 175-182. Sanya, China.

Takamatsu, D., Hirano, T., Yoneyama, A., Kimura, T., Harada, M., Terada, M. \& Okoshi, T. (2020). Chem. Commun. 56, 9553-9556.

Takamatsu, D., Yoneyama, A., Asari, Y. \& Hirano, T. (2018). J. Am. Chem. Soc. 140, 1608-1611.

Takeda, T., Yoneyama, A., Wu, J., Thet-Thet-Lwin, Momose, A. \& Hyodo, K. (2012). J. Synchrotron Rad. 19, 252-256.

Takeya, S. (2006). Appl. Phys. Lett. 88, 074103.

Takeya, S., Yoneyama, A., Ueda, K., Mimachi, H., Takahashi, M., Sano, K., Hyodo, K., Takeda, T. \& Gotoh, Y. (2012). J. Phys. Chem. $C$, 116, 13842-13848.

Thet Thet, L., Yoneyama, A., Imai, M., Maruyama, H., Hyodo, K. \& Takeda, T. (2018). Acta Radiologica Open, 7, 205846011880665.

Weitkamp, T., Diaz, A., David, C., Pfeiffer, F., Stampanoni, M., Cloetens, P. \& Ziegler, E. (2005). Opt. Express, 13, 6296-6304.

Weitkamp, T., Haas, D., Wegrzynek, D. \& Rack, A. (2011). J. Synchrotron Rad. 18, 617-629.

Yamada, S., Nakashima, T., Hirose, A., Yoneyama, A., Takeda, T. \& Takakuwa, T. (2012). Developmental Anatomy of the Human Embryo - 3D-Imaging and Analytical Techniques. in The Human Embryo, pp. 111-126. InTechOpen.

Yashiro, W., Kamezawa, C., Noda, D. \& Kajiwara, K. (2018). Appl. Phys. Express, 11, 122501.

Yashiro, W., Terui, Y., Kawabata, K. \& Momose, A. (2010). Opt. Express, 18, 16890-16901.

Yoneyama, A., Amino, N., Mori, M., Kudoh, M., Takeda, T., Hyodo, K. \& Hirai, Y. (2006). Jpn. J. Appl. Phys. 45, 1864-1868.

Yoneyama, A., Baba, R., Hyodo, K. \& Takeda, T. (2015). Eur. Congr. Radiol. 2015, C-0531.

Yoneyama, A., Baba, R., Hyodo, K., Takeda, T., Nakano, H., Maki, K., Sumitani, K. \& Hirai, Y. (2016). AIP Conf. Proc. 1696, 020007.

Yoneyama, A., Baba, R. \& Kawamoto, M. (2021). Opt. Mater. Express, 11, 398-411.

Yoneyama, A., Baba, R., Sumitani, K. \& Hirai, Y. (2015). Appl. Phys. Lett. 106, 084102.

Yoneyama, A. \& Kawamoto, M. (2020). J. Instrum. 15, P12029.

Yoneyama, A., Lwin, T. T. \& Kawamoto, M. (2020). J. Synchrotron Rad. 27, 468-471.

Yoneyama, A., Nambu, A., Ueda, K., Yamada, S., Takeya, S., Hyodo, K. \& Takeda, T. (2013). J. Phys. Conf. Ser. 425, 192007.

Yoneyama, A., Wu, J., Hyodo, K. \& Takeda, T. (2008). Med. Phys. 35, $4724-4734$ 